\title{
Bias-free time-integrating optical correlator using a photorefractive crystal
}

\author{
Demetri Psaltis, Jeffrey Yu, and John Hong
}

\begin{abstract}
An acoustooptic time-integrating correlator is demonstrated using a photorefractive crystal as the timeintegrating detector.
\end{abstract}

\section{Introduction}

Time integration ${ }^{1}$ has proved to be a powerful technique in optical signal processing and has been used in a wide variety of architectures. A major drawback of time-integrating processors is the buildup of bias in addition to the signal. This occurs because the photogenerated charge that is integrated on the detector is proportional to the intensity of the optical signal which makes it necessary to represent bipolar signals on a bias. The effective system dynamic range at the output is given by $\mathrm{DR}^{\prime}=\mathrm{DR}[\mathrm{SBR} /(1+\mathrm{SBR})]$ where $\mathrm{DR}$ is the dynamic range of the output detector and SBR is the signal-to-bias ratio on the detector. ${ }^{2}$ In most cases of interest, the SBR is much smaller than unity and thus the added bias significantly reduces the usable dynamic range of the system.

The most frequently used method for separating the signal from the bias involves placing the signal on a spatial carrier and then electronically filtering the output of the integrator. This method of bias removal, however, does not solve the dynamic range problem since the processing is done after the detection of the signal. Also, an additional constraint is placed on the resolution of the detector, since the pixel separation must be less than one-half of the period of the carrier being recorded, which will result in a significant reduction in the available space-bandwidth product at the output.

In this paper a new method for performing timeintegrating correlation is described using a photorefractive bismuth silicon oxide (BSO) crystal as the time-integrating element. The correlation is formed

\footnotetext{
The authors are with California Institute of Technology, Department of Electrical Engineering, Pasadena, California 91125.

Received 20 June 1985.

0003-6935/85/223860-06\$02.00/0.

(C) 1985 Optical Society of America.
}

on a spatial carrier in the crystal and read out with an auxiliary beam. Since only the signal recorded on a spatial carrier is stored in the photorefractive crystal, the diffracted light that is detected contains the correlation information without the bias. The bias does not reduce the dynamic range of the output detector used for final readout, but rather the diffraction efficiency of the BSO crystal. In addition, the resolution of the BSO crystal is very much higher than that of a CCD detector, allowing the correlation of very high space-bandwidth signals to be formed on a carrier. Finally, since the result of the time-integrating correlator is read out optically, the output can be easily interfaced with other optical systems, thus making new architectural designs possible.

In Sec. II, the theory of optical recording in photorefractive crystal is reviewed and extended to the use of photorefractive crystals as time-integrating elements. The architecture and experimental results are described in Sec. III. Dynamic range, linearity, system limitations, and other performance aspects are discussed in Sec. IV.

\section{Photorefractive Crystals as Time-Integrating Optical Detectors}

When a photorefractive BSO crystal is illuminated by an intensity grating, electrons are excited from traps into the conduction band. These charges migrate due to diffusion and drift from an externally applied electric field and then recombine in dark regions, creating a spatially varying internal space-charge field. This field modifies the index of refraction in the crystal through the linear electrooptic effect and, as a result, a holographic phase grating is recorded in the crystal. Grating formation in photorefractive media has been extensively studied and modeled..$^{3,4}$ We will show here that the photorefractive crystal acts as a time-integrating element.

Let the intensity incident on the crystal be as follows: 


$$
I(x, t)=\left\{\begin{array}{l}
I_{0}+\operatorname{Re}\left[I_{1}(x, t) \exp (i k x)\right] \text { for } t>0 \\
0 \text { otherwise }
\end{array}\right.
$$

Assuming that self-diffraction effects are negligible and that the spatial variations of $I_{1}(x, t)$ are small compared to the grating frequency $k$, the intensity of the light that is diffracted when the crystal is illuminated by a readout beam can be shown to be $^{5}$

$$
I_{\text {out }}(x, t)=\left|\frac{K_{1}}{\tau} \exp (-t / \tau) \int_{0}^{t} \frac{I_{1}\left(x, t^{\prime}\right)}{I_{0}} \exp \left(t^{\prime} / \tau\right) d t^{\prime}\right|^{2} I_{R} .
$$

$I_{R}$ is the intensity of the readout beam and $K_{1}$ is a complex constant involving the material parameters of the crystal, the grating frequency, and the applied electric field. $\tau$ is the complex time constant of the space-charge field and is given by ${ }^{3} \tau=K_{2} / I_{0} . K_{2}$ is also a complex constant that depends on the photorefractive material used and the experimental conditions. $I_{0}$ is the average light intensity incident on the crystal during exposure.

If $I_{1}(x, t)$ is expanded into its temporal Fourier components,

$$
I_{1}\left(x, t^{\prime}\right)=\int_{-\infty}^{\infty} \tilde{I}_{1}(x, w) \exp \left(i w t^{\prime}\right) d w
$$

then the output intensity for $t \gg \tau$ can be written as follows:

$$
I_{\text {out }}(x, t) \cong\left|\frac{K_{1}}{\tau I_{0}} \int_{-\infty}^{\infty} \frac{\tilde{I}_{1}(x, w)}{(1 / \tau+i w)} \exp (i w t) d w\right|^{2} I_{R} .
$$

The above is recognized to be a low pass filter with cutoff frequency $1 /|\tau|$, which is approximately equivalent to the output of a sliding window integrator, with integration time $\tau$. Thus,

$$
I_{\text {out }}(x, t) \cong\left|\frac{K_{1}}{\tau} \int_{t}^{t+\tau} \frac{I_{1}\left(x, t^{\prime}\right)}{I_{0}} d t^{\prime}\right|^{2} I_{R}
$$

Hence, the output intensity can be treated as the square of the normalized integration of the signal $I_{1}$. An interesting observation is that, if $I_{1}(x, t)$ were independent of time, the output intensity depends only on the ratio of the modulated intensity $I_{1}$ to the dc intensity $I_{0}$.

\section{Experimental Demonstration}

A schematic diagram of the experimental system is shown in Fig. 1. The input electrical signals are mixed with the center frequency of the acoustooptic devices (AODs) and fed into the AODs. The first AOD is illuminated by a collimated wave and the upshifted diffracted order is imaged onto the second AOD, then reimaged onto the photorefractive BSO crystal. The second AOD is oriented such that the acoustic signal is counterpropagating with respect to the image of the acoustic signal from the first AOD. The undiffracted light transmitted through the first AOD is incident at the Bragg angle of the second AOD. The upshifted diffracted order of the second AOD is also imaged onto the BSO crystal. The undiffracted light is spatially filtered before reaching the BSO crystal. In this arrangement, the AODs are parallel to each other, but the diffracted orders propagate at an angle with respect to each other even though both diffracted beams are temporally upshifted. This causes the signals from the two AODs to interferometrically record the correlation signal on the BSO crystal at a high spatial frequency. Let the inputs to the AODs be $v_{1}(t)=a(t)$ $\exp \left(i w_{0} t\right)$ and $v_{2}(t)=b(t) \exp \left(i w_{0} t\right)$, where $w_{0} / 2 \pi$ is the center frequency of the AOD. The intensity incident on the photorefractive crystal is

$$
\begin{aligned}
I(x, t) & =|a(t-x / v) \exp (i \gamma x)+b(t+x / v) \exp (-i \gamma x)|^{2} \\
& =|a(t-x / v)|^{2}+|b(t+x / v)|^{2} \\
& +2 \operatorname{Re}\left[a(t-x / v) b^{*}(t+x / v) \exp (i 2 \gamma x)\right],
\end{aligned}
$$

where $v$ is the acoustic velocity of the AOD and $\gamma=$ $w_{0} / v$. We will treat the case where $|a(t)|^{2}$ and $|b(t)|^{2}$ can both be approximated as constants, as is the case for FM signals. Then, the intensity pattern can be separated into a dc term $I_{0}$ and a signal term $I_{1}(x, t)$ modulating a spatial carrier $\cos (2 \gamma x)$ in the form of $\mathrm{Eq}$. (1). This intensity pattern results in the formation of a hologram on the photorefractive crystal as described in the previous section. The hologram is read out with an auxiliary beam and is imaged onto a charge coupled device (CCD) detector for readout.

If the assumption is valid that $I_{1}(x, t)$ has spatial frequencies which are small compared with the carrier frequency $2 \gamma$, we can use Eq. (4) to obtain an expression for the output intensity detected by the CCD:

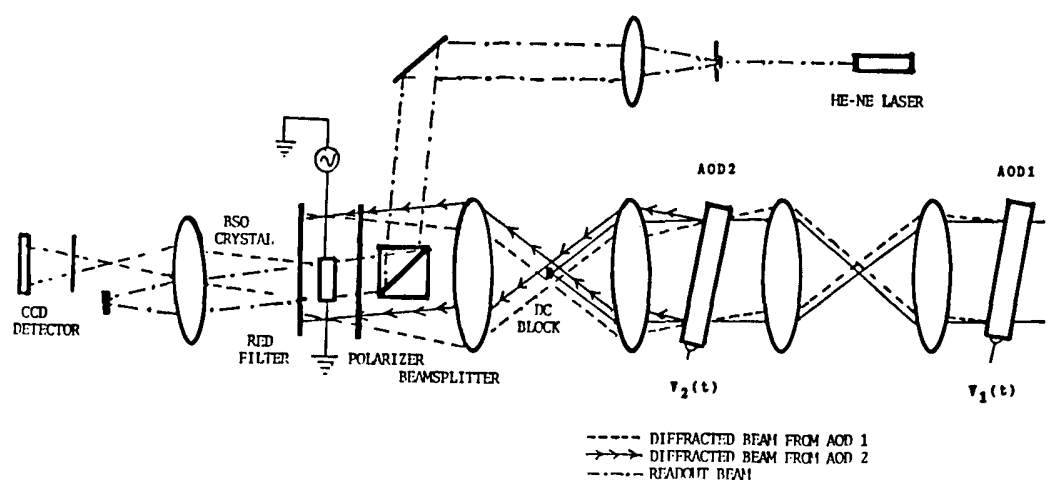

Fig. 1. Optical setup of the photorefractive bias removal correlator. 


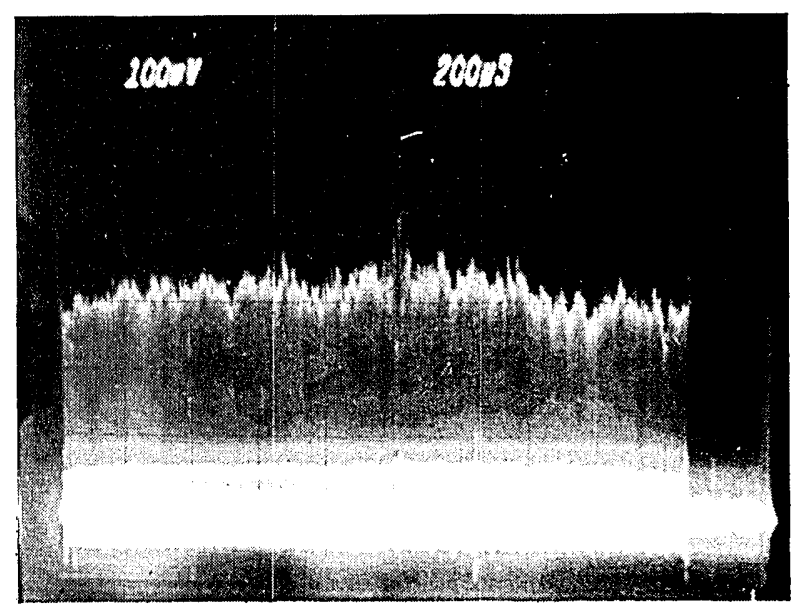

Fig. 2. Output of a standard time-integrating correlator without noise.

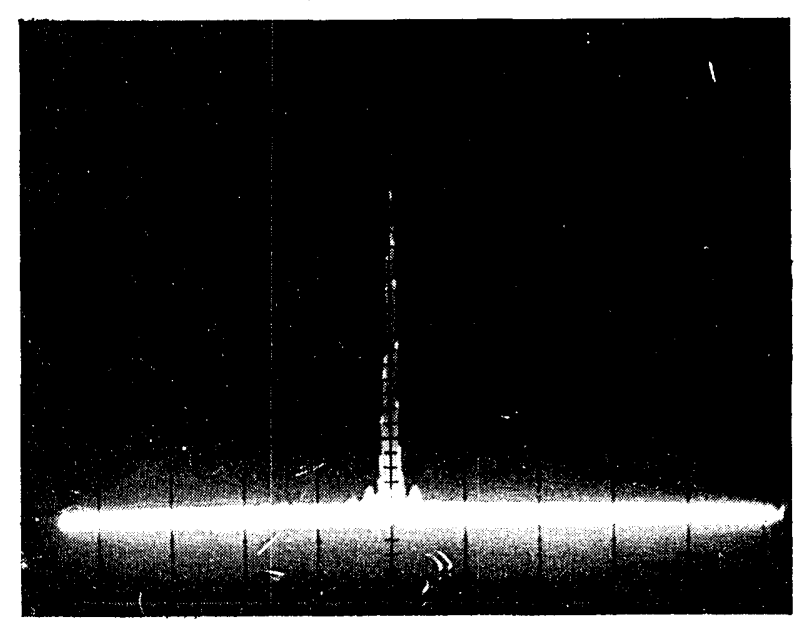

Fig. 3. Output of the bias removal correlator without noise.

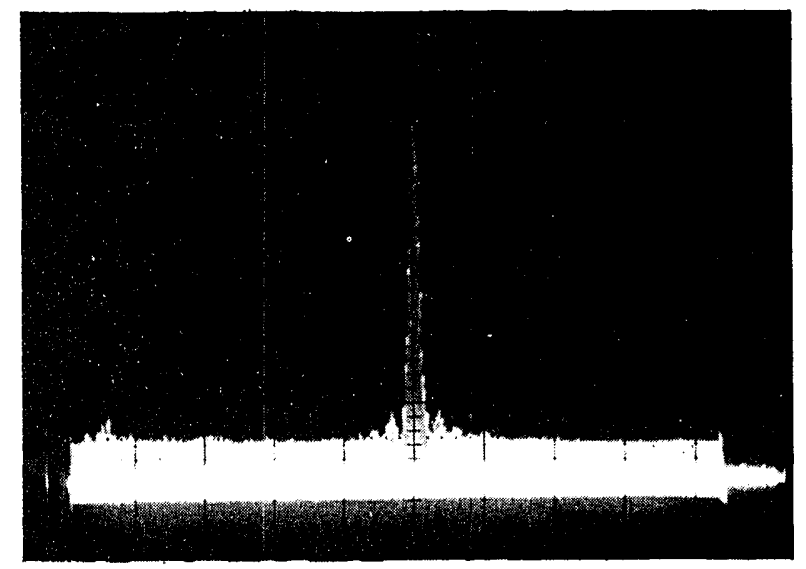

Fig. 4. Output of the bias removal correlator with a signal-to-noise ratio of $0 \mathrm{~dB}$.

$$
I_{\text {out }}(x) \cong I_{R}\left|\frac{K_{1}}{\tau}\right|^{2}\left|\int_{t}^{t+\tau} \frac{a(t-x / v) b^{*}(t+x / v)}{|a|^{2}+|b|^{2}} d t\right|^{2},
$$

and by defining variable $t_{1}=t-x / v$,

$$
I_{\text {out }}(x) \propto\left|\int_{t+x / v}^{t+\tau-x / v} a\left(t_{1}\right) b^{*}\left(t_{1}+2 x / v\right) d t_{1}\right|^{2} .
$$

Hence the system produces the magnitude square of the correlation between the signals $a(t)$ and $b(t)$ integrated over a finite interval $r$.

Flint glass acoustooptic cells driven at a center frequency of $70 \mathrm{MHz}$ were used in the experiment. A symmetric linear chirp signal with bandwidth $\Delta f=5$ Mhz was fed into each cell to produce the autocorrelation peak. The Bragg angle of the AODs was $0.2^{\circ}$, which corresponded to a grating frequency equal to 35 lines/mm in the BSO crystal.

The BSO crystal used in the experiment was cut in the $\langle 110\rangle$ direction and measured $15 \times 15 \times 2 \mathrm{~mm}$. An external electric field of $7 \mathrm{kV} / \mathrm{cm}$ was applied in the $\langle 001\rangle$ direction of the crystal which was also the direction of the grating vector.

The correlation was recorded on the crystal with an argon laser at a wavelength of $514 \mathrm{~nm}$ with average intensity equal to $1 \mu \mathrm{W} / \mathrm{cm}^{2}$. The correlation was read out with a He-Ne laser $(\lambda=633 \mathrm{~nm})$ with $150-\mu \mathrm{W} / \mathrm{cm}^{2}$ intensity. Cylindrical lenses (not shown in Fig. 1) were used to expand the output of the AODs thereby illuminating the full aperture of the BSO crystal and also to focus the diffracted light onto a 1-D CCD.

The output signal-to-bias ratio of a conventional time-integrating correlator is reduced when the levels of the two signals are unequal and/or if there is additive noise present in the system. Both conditions were simulated experimentally. Noise was simulated by adding a $70-\mathrm{MHz}$ signal to the input of one of the AODs. The output of a standard time-integrating correlator (i.e., the correlation formed directly on the CCD) for the noise-free case and equal amplitude signals is shown in Fig. 2. This condition provides the maximum signal-to-bias ratio for the system. We can

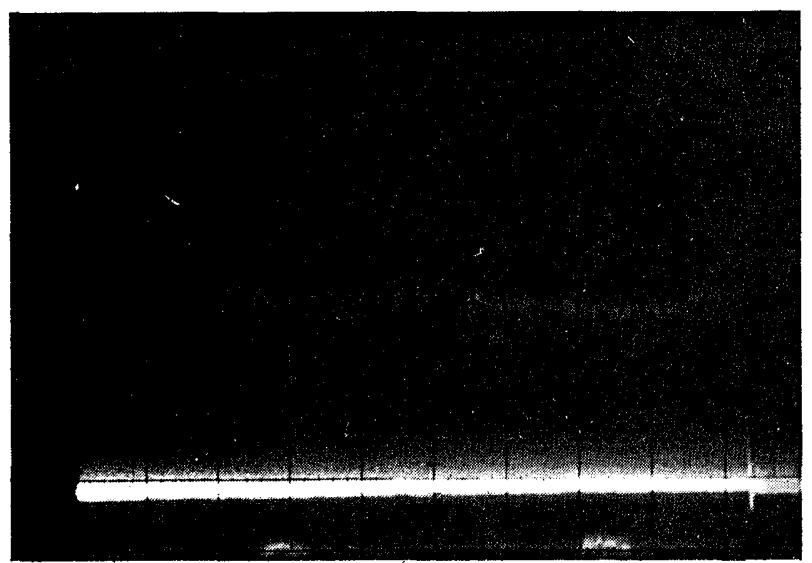

Fig. 5. Output of the bias removal correlator with a signal-to-noise ratio of $-10 \mathrm{~dB}$. 
see in Fig. 2 that there is still a strong bias term added to the correlation peak. The correlation produced by temporally integrating on the photorefractive crystal is shown in Fig. 3. In this case, all the bias due to temporal integration is removed, and any residual bias is due entirely to dark current from the CCD. The outputs of the bias removal correlator with input signal-to-noise ratios of 0 and $-10 \mathrm{~dB}$ are shown in Figs. 4 and 5, respectively. Again, bias levels which appear in the figures were entirely due to the integration of dark current in the output detector. In practice, the detector dark current can be minimized by increasing the intensity of the readout beam, thereby decreasing the required integration time of the output CCD detector and/or cooling the detector.

\section{Performance}

The experimental results described in the previous section show a dramatic qualitative improvement in the correlation that is obtained when the photorefractive crystal is used instead of the CCD. In this section we examine certain characteristics of this method which are useful for quantitatively evaluating its performance. Specifically, we examine the linearity, integration time, dynamic range, and sensitivity of the correlator.

\section{A. Linearity}

In a conventional time-integrating correlator (coherent or incoherent), the output correlation is basically proportional to the signals applied to the AODs. Nonlinearities occur only when we exceed the linear dynamic range of the devices used, i.e., if the diffraction efficiency of the AOD exceeds several percent or the integrating detector is driven to saturation. In the photorefractive time-integrating processor, the output intensity is a nonlinear, monotonically increasing function of the input voltage. The nonlinearity arises because of the square-law detection at the final readout stage and the recording mechanism in the photorefractive crystal. The nonlinear relationship is now studied analytically and experimental verification of the theoretical results is presented.

Let $v_{1}(t)=s(t)$ be a fixed reference signal and $v_{2}(t)=$ $a s(t)$ be an input signal of varying amplitude $(0<a<$ $1)$. Since the correlation term contains spatial frequencies which are much lower than the grating frequency, near the correlation peak $(x=0)$ the intensity incident on the photorefractive crystal is

$$
I(x, t)=\left(1+a^{2}+2 a \cos k x\right)|s(t)|^{2} .
$$

Using Eq. (1), the output intensity at the CCD is proportional to

$$
I_{\text {out }} \propto\left|\frac{2 a}{1+a^{2}} \tau\right|^{2} .
$$

The modulation depth of the intensity incident on the BSO crystal is

$$
m=\frac{2 a}{1+a^{2}},
$$

and hence

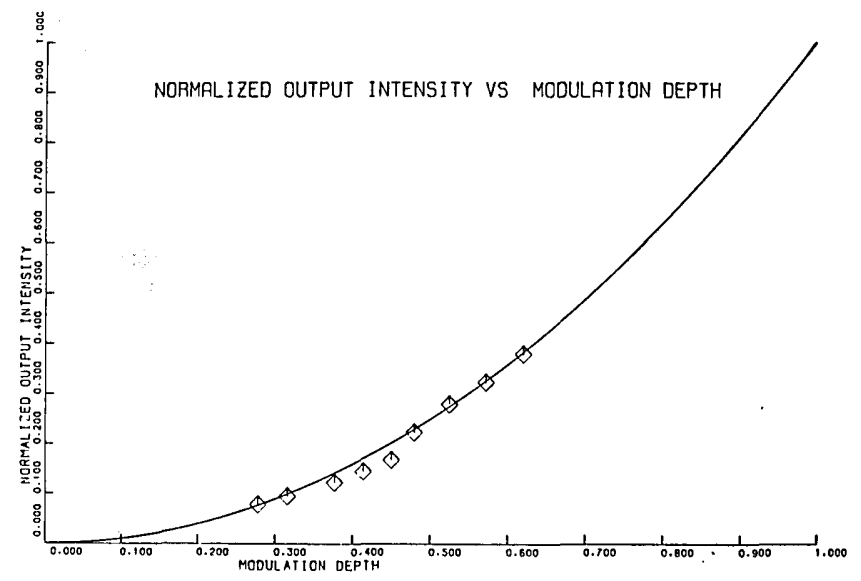

Fig. 6. Normalized output intensity vs modulation depth.

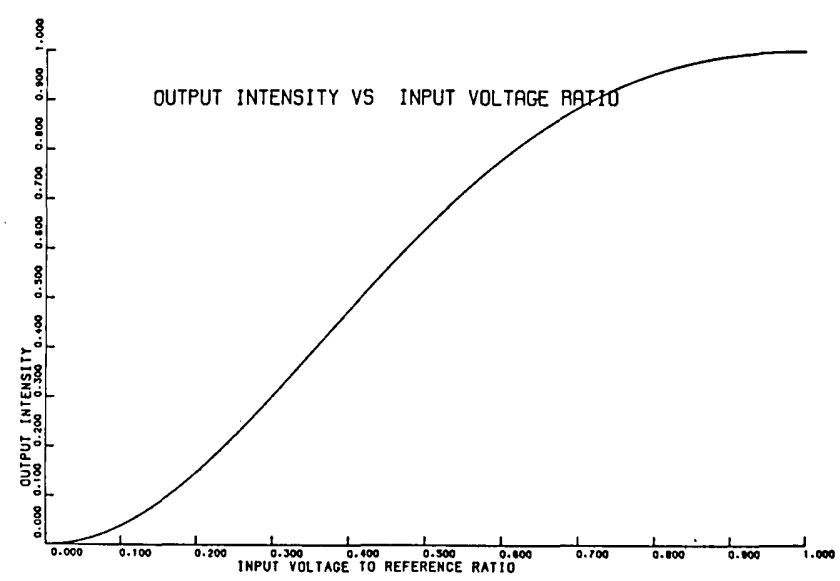

Fig. 7. Theoretical plot of output intensity vs input voltage ratio.

$$
I_{\text {out }} \propto m^{2}=4 a^{2} /\left(1+a^{2}\right) .
$$

Figure 6 is a graph of the output intensity at the correlation peak vs the modulation depth incident on the crystal. The experimental result is in excellent agreement with the square-law relationship predicted by Eq. (7).

A plot of the output intensity as a function of the amplitude of the input signal $a$ is shown in Fig. 7. The nonlinear relationship between the input and output signals is generally a disadvantage since the scaling of signals of varying amplitudes will be nonlinear. This, however, will not cause a problem if the correlator is used only as a signal detection device, since correlation peaks will still be discernible and only the threshold level need be adjusted accordingly to maximize the probability of detection.

\section{B. Integration Time}

In a conventional time-integrating correlator, the integration time is limited by the dark current buildup on the output detector, typically up to several hundred milliseconds. When the photorefractive crystal is 


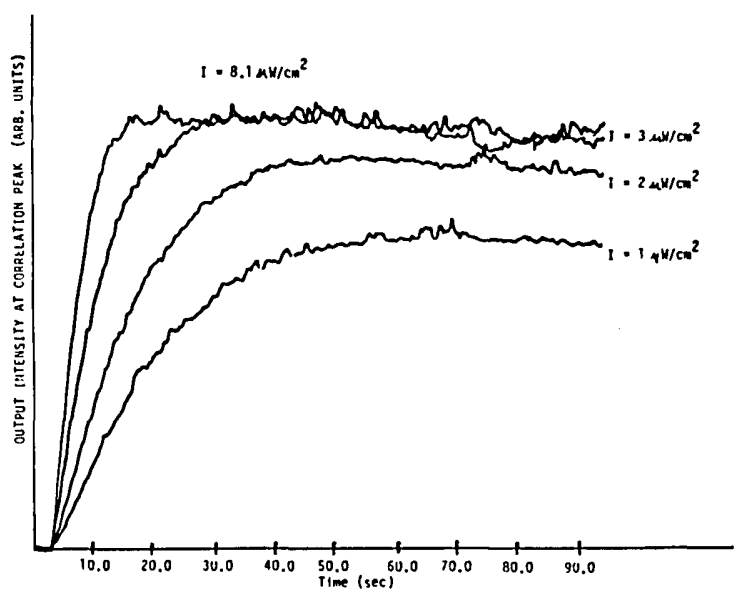

Fig. 8. Output intensity at correlation peak vs time as a function of different average incident intensities.

used, the integration time is determined by the rise time of the internal space-charge field which can easily be made much longer. The correlation can be read out at any rate that is convenient by an auxiliary detector array.

The integration time is approximately equal to $|\tau|$, where

$$
|\tau|=\left|\frac{K_{2}}{I_{0}}\right|
$$

Hence, the integration time of the bias removal correlator can be controlled by varying the writing intensity. This control is important since the integration time can be matched to the length of the reference signal thereby increasing the probability of detection of a weak signal.

The time response of the correlation peak for different values of average incident intensity is shown in Fig. 8. Figure 9 is a plot of intensity vs the inverse of the experimentally observed rise time. There is excellent agreement between the experiment and Eq. (8).

The integration time, however, has a finite range over which it can be adjusted. The maximum integration time is limited by the thermal effects in the crystal. If the rate at which carriers are generated thermally becomes comparable with the rate at which they are photogenerated, the modulation depth of trap density will be reduced. As a result, the diffraction efficiency of the grating will decrease. In practice, the minimum integration time is limited by the maximum light intensity that is available for recording. The integration time can be reduced to $30 \mathrm{msec}$ if the incident intensity is made equal to $18 \mathrm{~mW} / \mathrm{cm}^{2}$. This power level, however, is simply not practical for most applications.

\section{Dynamic Range and Sensitivity}

Since the output of the bias removal correlator is presented without bias, the output dynamic range of the system is essentially equal to the dynamic range of the readout detector array. To characterize the per-

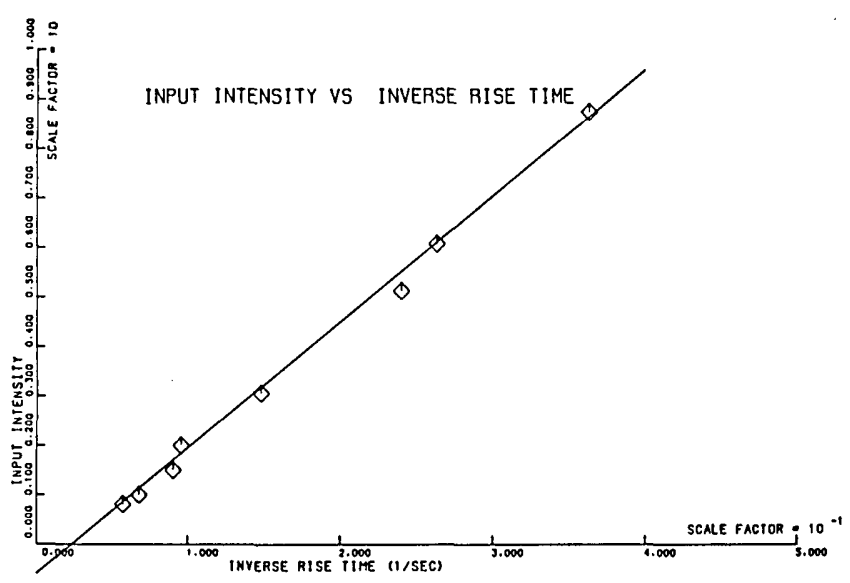

Fig. 9. Inverse of the rise time vs average incident intensity.

formance of the system we need to determine how the input signal levels are mapped to this output dynamic range. Let the dynamic range of the photorefractive crystal be defined as $\mathrm{DR}_{\mathrm{BSO}}=m_{\max } / m_{\min }$, where $m_{\max }$ is the maximum modulation depth $\left(m_{\max }=1\right)$, and $m_{\min }$ is the minimum modulation depth for which a diffracted signal is detectable above the output scatter and noise level of the system.

Given two input signals $v_{1}(t)=a s(t)$ and $v_{2}(t)=s(t)$, the modulation depth of the light incident on the crystal is $m=2 a /\left(a^{2}+1\right)$. Thus, the minimum detectable input signal is given by $a_{\min }=m_{\min } / 2=1 / \mathrm{DR}_{\mathrm{BSO}}$. The useful range over which $a$ can vary is limited by $\mathrm{DR}_{\mathrm{BSO}}$. From $a_{\min }$, one can define an input dynamic range given by $\mathrm{DR}_{\text {input }}=1 / a^{2} \min =4 / \mathrm{m}^{2}$ min. The most important parameter in determining the system dynamic range is the minimum detectable modulation depth $m_{\mathrm{min}}$. Experimentally, we measured the dynamic range to be equal to $23 \mathrm{~dB}$. This corresponds to a minimum modulation depth of 0.142 . We expect that through careful design this can be substantially improved. However, all the mechanisms that determine $m_{\min }$ are not fully understood. It is believed that besides detector noise and scattering from the crystal, the modulation depth is limited by thermal effects in the material and shot noise arising from the internal currents.

Another important aspect of the correlator system is its sensitivity or the minimum signal-to-noise ratio that is detectable. This parameter is also determined by the minimum detectable modulation depth, $m_{\min }$. Given a reference signal $v_{1}(t)=a s(t)$ and an input signal contaminated by additive noise, $v_{2}(t)=b s(t)+$ $n(t)$, the modulation depth of the intensity incident on the crystal is

$$
m=\frac{2 a b|s(t)|^{2}}{\left(a^{2}+b^{2}\right)|s(t)|^{2}+{\sigma_{n}}^{2}} .
$$

The reference level which maximizes $m$ is given by $a=$ $\left(b^{2}+\sigma_{n}^{2} /|s(t)|^{2}\right)^{1 / 2}$, corresponding to a modulation depth of 


$$
m=\frac{b}{\left(b^{2}+\sigma_{n}^{2} /|s(t)|^{2}\right)^{1 / 2}} .
$$

In practice, optimizing the reference level can easily be achieved by setting the power of the reference equal to the total average power of the input signal.

Normalizing the signal and noise terms such that $|s(t)|^{2}={\sigma_{n}}^{2}=1$ we obtain

$$
m=\frac{b}{\left(b^{2}+1\right)^{1 / 2}} .
$$

Thus, the minimum input SNR that produces a detectable correlation peak at the output is $(\mathrm{S} / \mathrm{N})_{\min }=$ $\left(b^{2} / \sigma_{n}{ }^{2}\right)_{\min } \cong m_{\min 2}$.

From the experimentally measured value of $m_{\min }$, the correlator should have had a sensitivity of $-17 \mathrm{~dB}$. However, experimental results showed a sensitivity of $-14 \mathrm{~dB}$.

\section{Conclusion}

The photorefractive time-integrating processor that has been described has several advantageous features: bias removal, increase in the output space-bandwidth product, and the ability to directly interface the result of the time-integrating processor with other optical systems. Bias-free correlation is desirable because it allows us to increase the dynamic range and hence the sensitivity of time-integrating processors. In the implementation described in this paper, however, the square-law detection at the output reduces the available overall dynamic range. A definite improvement in dynamic range can be obtained if the correlation that is formed in the photorefractive crystal is interferometrically detected on the output detector. Another limitation of the system described here is the long integration time (several seconds). In some applications this long integration time is desirable and could result in extremely good sensitivity (detection of signals with very low SNR). However it is certainly desirable to be able to decrease the integration time to several milliseconds. This could be accomplished by increasing the optical power of the writing beams, but this is in general an impractical solution. Another limitation of this technique is the relatively low diffraction efficiency that is obtained with BSO crystals (2-3\%), which reduces the overall light efficiency. Materials with higher electrooptic coefficients, such as barium titanate, can provide better efficiency; however, the time constant obtained with this particular material is much longer than that of BSO. New photorefractive materials currently being developed showing promise of a large improvement in optical sensitivity as well as higher electrooptic coefficients may provide a substantial improvement in performance and, specifically, reduce the total optical power that is required.

This work is supported by the Air Force Office of Scientific Research and the Army Research Office.

\section{References}

1. R. A. Sprague and C. L. Koliopoulos, "Time Integrating Acoustooptic Correlator," Appl. Opt. 15, 89 (1976).

2. D. Psaltis, "Incoherent Electrooptic Image Correlator," Opt. Eng. 23, 12 (1984).

3. N. V. Kukhtarev, V. B. Markov, S. G. Odulov, and M. S. Soskin, "Holographic Storage in Crystals. I: Steady State," Ferroelectrics 22, 949 (1979).

4. J. Feinberg, D. Heiman, A. R. Tanguay, and R. W. Hellwarth, "Photo Refractive Effects and Light-Induced Charge Migration in Barium Titanate," J. Appl. Phys. 51, 1297 (1980).

5. M. Cronin-Golomb, "Large Nonlinearities in Four-Wave Mixing in Photorefractive Crystals and Applications in Passive Optical Phase Conjugation," Ph.D. Thesis, California Institute of Technology (Mar. 1983).

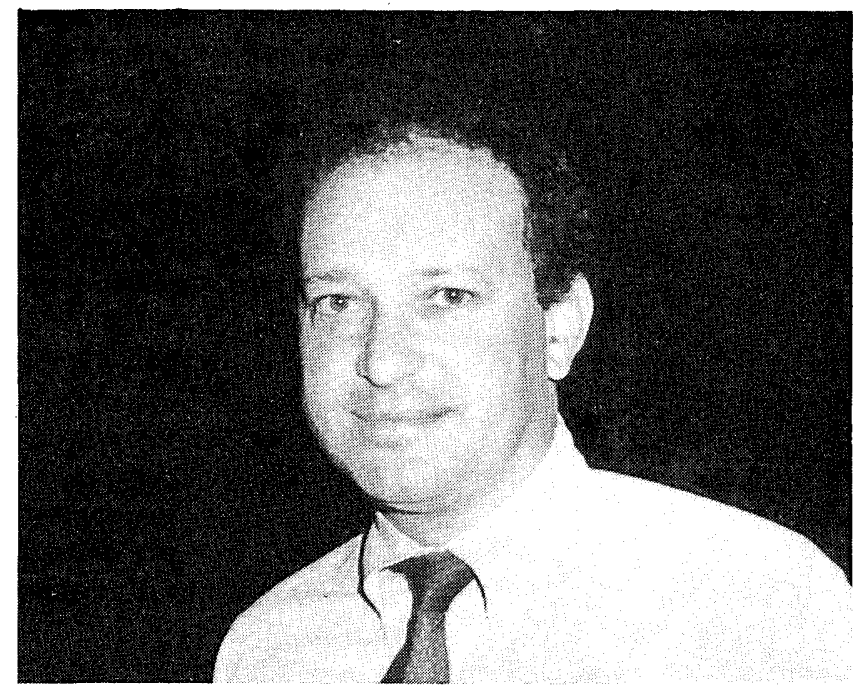

John Moharam of Georgia Tech at the 1984 OSA Annual Meeting. Photo: F. S. Harris, Jr. 